Wahl 2008 gezogen. Zwar gab es weiterhin Wunschkoalitionen - Rot-Grün, SchwarzGelb -, aber zugleich Offenheit für andere Konstellationen. „Ausschließeritis“ kam kaum noch vor.

(4) Die CDU ging als klar stärkste Kraft aus der Landtagswahl hervor; die SPD konnte nach ihrem desaströsen Ergebnis 2009 um sieben Prozentpunkte zulegen. Die Grünen erzielten - für sie enttäuschende - 11,1 Prozent. FDP und Linke konnten mit knapp über fünf Prozent in den Landtag einziehen. Die Wahlbeteiligung lag bei 73,2 Prozent und damit um 12,2 Prozentpunkte höher als bei der Wahl zuvor - eine unmittelbare Folge der Zusammenlegung der Landtags- mit der Bundestagswahl.

(5) Das Wahlergebnis verschaffte keiner der beiden Wunschkoalitionen eine Mehrheit. Auf die Wahl folgte daher eine lange Phase von Sondierungsgesprächen zwischen CDU und Grünen, CDU und SPD sowie zwischen SPD, Grünen und Linken. Nachdem Gespräche über ein Linksbündnis früh ins Stocken geraten waren, entschied sich die Union letztlich, den Grünen Koalitionsverhandlungen anzubieten. Die Grünen nahmen dieses Angebot an, und die eigentlichen Koalitionsverhandlungen wurden zügig abgeschlossen. Im Januar 2014 wurde Volker Bouffier im Hessischen Landtag in seinem Amt als Ministerpräsident bestätigt.

\title{
Klarmachen zum Ändern? Die Piratenfraktion nach zwei Jahren im Berliner Abgeordnetenhaus
}

\author{
Carsten Koschmieder
}

Nachdem die Piratenpartei bei der Wahl am 18. September 2011 mit 8,9 Prozent der Stimmen fünfzehn Mandate im Berliner Abgeordnetenhaus errungen hatte, gab es in der Öffentlichkeit, vor allem aber bei ihren Wählern und Anhängern die Erwartung, diese in so vielen Punkten ungewöhnliche Partei würde auch im Parlament alles ,anders“ machen. Der Spitzenkandidat Andreas Baum antwortete denn auch auf die Frage, worauf er in fünf Jahren besonders stolz sein werde: „Dass sich durch die Piraten im Abgeordnetenhaus viel verändert hat. "I Ihre Versprechen und ihre Rhetorik im Wahlkampf ließen in der Tat viele Veränderungen erwarten: Die stärkere Beteiligung der Bürger an den Entscheidungen des Parlaments, eine neue Kultur der Transparenz, die Ablehnung der Fraktionsdisziplin sowie ein freches, unangepasstes Auftreten - auf all diese Ideen bezogen sich Aussagen wie die des frischgebackenen Abgeordneten Simon Weiß, der auf der Wahlparty sagte: „Wir wollen Politik anders machen." ${ }^{2}$ Nach der Wahl allerdings geriet die neugebildete Fraktion schnell in das Spannungsfeld zwischen ihren eigenen Vorstellungen von Politik einerseits, den Anforderun-

1 Jens Anker, „Das Experiment hat sich gelohnt“, in: Berliner Morgenpost vom 4. September 2011, S. 2.

2 Karin Christmann, Wir werden von uns hören lassen, in: Der Tagesspiegel vom 19. September 2011, S. 5. 
gen und Zwängen der Arbeit im Abgeordnetenhaus andererseits. Auch der äußere und innere Druck auf die Fraktion, sich zu professionalisieren und der Logik der Mediendemokratie anzupassen, stellte ihre unkonventionelle Arbeitsweise von Beginn an in Frage. Nach gut zwei Jahren im Abgeordnetenhaus ist es angebracht, zu fragen, inwieweit sich die Fraktion der Piratenpartei in den Bereichen Transparenz, Basisbeteiligung, Hierarchie und Fraktionsdisziplin sowie in ihrem Auftreten zunächst tatsächlich von den „etablierten“ Parteien unterschied, wie sich diese Unterschiede entwickelt haben und worauf dies zurückzuführen ist.

Die Gründe, gerade die Berliner Piratenfraktion zu untersuchen, liegen auf der Hand: Zwar folgten dem Erfolg in Berlin der Einzug in drei weitere Landesparlamente, nämlich im Saarland, in Schleswig-Holstein und in Nordrhein-Westfalen; den dortigen Fraktionen diente die Erfahrung der Berliner Piraten aber bereits einerseits als Vorbild, andererseits als Mahnung, welche Fehler sich vermeiden ließen. ${ }^{3}$ Innerhalb der Piratenpartei besitzt die Berliner Fraktion als die erste also eine besondere Stellung, ihre Mitglieder gelten als einflussreich. ${ }^{4}$ Vor allem aber lassen sich durch die mittlerweile erreichte Halbzeit im Berliner Parlament die möglichen Veränderungen wesentlich besser ermitteln als bei jüngeren Fraktionen.

Trotz dieser Beschränkung weist die Untersuchung durchaus über die Berliner Landespolitik und auch über die Piratenpartei hinaus. Die Kritik an der Funktions- und Arbeitsweise des von Parteien dominierten parlamentarischen Systems, gegen die sich die Änderungswünsche der Piraten richten, ist schließlich keineswegs neu, sondern lässt sich vielmehr von Carl Schmitt bis Jürgen Habermas in unterschiedlichsten Varianten finden. ${ }^{5}$ Die Frage, inwieweit die Ideen der Piraten in der Praxis umsetzbar und damit geeignet sind, das Parlament ein Stück weit zu verändern, ist daher für die Parlamentarismusforschung insgesamt von Bedeutung.

Zur Beantwortung der drei Leitfragen sollen im Folgenden die verschiedenen Felder analysiert werden, in denen sich die Piratenpartei zunächst von den „etablierten“ Parteien unterschied. Dabei werden die Bereiche untersucht, in denen die Piratenfraktion bewusst qualitativ etwas anders machen wollte, nicht aber solche, in denen die Partei sich lediglich aus strukturellen Gründen oder fehlender Erfahrung unterscheidet, oder jene, in denen sie sich beispielsweise durch besonders gute oder besonders schlechte inhaltliche Arbeit abhebt. Außerdem muss eine umfassende Analyse der gesamten Arbeit der Fraktion, auf deren Erkenntnisse auch die hier getroffene Auswahl zurückgeht, an anderer Stelle erfolgen. ${ }^{6}$ Generell ist die Arbeit der Fraktionen der Piratenpartei in den Landesparlamenten bisher wenig erforscht, lediglich die umfassende Studie von Alexander Hensel und Stephan Klecha enthält ein Kapitel über alle vier Piratenfraktionen. ${ }^{7}$ Daher stützt sich der Artikel hauptsächlich auf Primärquellen wie Sitzungsprotokolle, Blogeinträge, Medienberichte oder teilnehmende Beobachtung beispielsweise bei Landesmitgliederversammlungen. Außerdem basiert er auf Interviews, die am 6. März 2012, am 17. und am 24. Juli 2013 sowie am 12. August 2013 mit den Abgeordneten Andreas Baum, Gerwald Claus-Brunner, Susanne Graf,

3 Vgl. Carsten Koschmieder, Die Piratenfraktion im Berliner Abgeordnetenhaus, in: Oskar Niedermayer (Hrsg.), Die Piratenpartei, Wiesbaden 2013, S. 213 - 235, S. 231.

4 Vgl. Astrid Geisler, Crowd und Rüben, in: taz vom 10. Mai 2013, S. 3.

5 Siehe dazu u. a. Hartmuth Becker, Die Parlamentarismuskritik bei Carl Schmitt und Jürgen Habermas, Berlin 1994.

6 Vgl. Carsten Koschmieder, a.a.O. (Fn. 3).

7 Vgl. Alexander Hensel / Stephan Klecha, Die Piratenpartei. Havarie eines politischen Projekts?, Frankfurt am Main 2013, S. 72 - 79. 
Heiko Herberg, Oliver Höfinghoff, Simon Kowalewski, Christopher Lauer, Wolfram Prieß und Fabio Reinhardt sowie der für Kommunikation und Koordination zuständigen Mitarbeiterin der Fraktion Monika Belz geführt wurden.

\section{Die Besonderheiten der Piratenfraktion}

Vor allem hinsichtlich Transparenz, Basisbeteiligung, Hierarchie und Fraktionsdisziplin sowie für ihr Auftreten im Parlament hatten sich die Piraten vorgenommen, viele Dinge anders zu handhaben als die Fraktionen anderer Parteien.

\subsection{Transparenz}

Mit wohl keinem Schlagwort hat die Piratenpartei so viel Aufsehen erregt, aber auch so viel Erfolg gehabt wie mit der „Transparenz“. Dabei bedeutet „Erfolg“ hier einerseits, dass die Forderung nach mehr Transparenz in der Politik entscheidend mit zum Wahlerfolg der Partei beigetragen hat $^{8}$, andererseits aber auch, dass innerhalb kürzester Zeit sämtliche Parteien (zumindest verbal) für mehr Transparenz eintraten und die Piraten so bereits „parteistrategische Relevanz für ihre Konkurrentinnen“9 gewannen. Im Berliner Wahlkampf hatten sie eine klare Position bei diesem Thema bezogen. So findet sich im Wahlprogramm die Forderung, „eine transparente Politik im Abgeordnetenhaus, in der Senatsverwaltung und in den Bezirksverwaltungen“ umzusetzen, da nur „durch transparente Politik und Verwaltung“ die Möglichkeit bestehe, „Rückschlüsse auf das Handeln der Ausschüsse, Verwaltungen und Abgeordneten zu ziehen“. Auch hätten aus Sicht der Piraten „die Altparteien kein Interesse an transparenter Politik"10. Die Bedeutung des Themas lässt sich daran ersehen, dass zwei Wochen vor der Wahl der Spitzenkandidat Andreas Baum auf die Frage, was ihm inhaltlich am wichtigsten sei, antwortete, das sei „natürlich das Thema Transparenz"11.

Gemäß ihren Aussagen im Wahlkampf startete die neugewählte Fraktion am Montag nach der Wahl mit einem öffentlichen Treffen, das nicht nur für Besucher zugänglich war, sondern dessen Protokoll auch im Internet veröffentlicht wurde. ${ }^{12}$ Dabei wurde neben vielen organisatorischen Dingen auch darüber gesprochen, wie der Transparenzanspruch bei den wöchentlichen Fraktionssitzungen in die Praxis umgesetzt werden könnte. Erstmals

8 Nach dem erfolgreichen Bürgerbegehren zur Offenlegung der Verträge der Teilprivatisierung der Wasserversorgung waren die Wähler für das Thema ohnehin sensibilisiert. Vgl. Oskar Niedermayer, Die Piraten im parteipolitischen Wettbewerb: von der Gründung Ende 2006 bis zu den Wahlerfolgen in Berlin 2011 und im Saarland 2012, in: ders. (Hrsg.), a.a.O. (Fn. 3), S. 29 - 62, S. 47 f.

9 Ders., Einleitung: Erfolgsbedingungen neuer Parteien im Parteiensystem, in: ebenda, S. 7 - 14, S. 9.

10 Das „Wahlprogramm Berlin 2011“ der Piratenpartei ist einsehbar unter http://www.piratenpartei.de/wp-content/uploads/2012/02/Wahlprogramm-Berlin-2011.pdf (Abruf am 27. Juni 2013). Allerdings fand sich auch schon im Wahlprogramm der Berliner Grünen an prominenter Stelle die Forderung nach mehr Transparenz in Politik und Verwaltung sowie bei den Abgeordneten. Vgl. Bündnis 90/Die Grünen, Eine Stadt für alle. Das Wahlprogramm zur Berliner Abgeordnetenhauswahl 2011, Berlin 2011, S. 20 ff., S. 173.

11 Jens Anker, a.a.O. (Fn. 1).

12 Vgl. Piratenpartei, Protokoll der Sitzung, http://piratenpad.de/kt7 (Abruf am 5. April 2012). 
zeichnete sich hier eine Konfliktlinie ab, die die Fraktion bis heute beschäftigt. Die Debatte entzündete sich vor allem an der Frage, wie - wenn überhaupt - die Öffentlichkeit für die Sitzungen erreicht werden sollte. Dem Vorschlag, dass die Öffentlichkeit grundsätzlich zugelassen sei und nur zum Schutz von Persönlichkeitsrechten beispielsweise bei Bewerbungen temporär ausgeschlossen werden könne und dass diese Öffentlichkeit auch über einen Livestream im Internet hergestellt werden müsse, widersprachen einige Abgeordnete. Heiko Herberg und Christopher Lauer führten die funktionellen Vorteile nichtöffentlicher Sitzungen ins Feld, da die Fraktionsmitglieder im Beisein der Presse nicht offen und unverstellt sprechen könnten. Pavel Mayer regte sogar an, die Sitzung aufzuzeichnen und bestimmte Stellen vor einer Veröffentlichung zu „zensieren“. Dieser für einen Piraten ungewöhnliche Vorschlag machte deutlich, dass weder in der Partei noch unter den Abgeordneten Einigkeit darüber herrschte, was unter dem Stichwort „Transparenz“ in der Praxis eigentlich zu verstehen sei und wie diese im politischen Alltag umgesetzt werden sollte. Insbesondere Gerwald Claus-Brunner erinnerte an die versprochene umfassende Transparenz und verlangte, dieses Versprechen nun auch einzuhalten. Als Kompromiss wurde schließlich vorgeschlagen, dass die Abgeordneten als Fraktion Transparenz herstellen müssten, als Gruppe von Menschen aber auch mal ohne (Internet-)Öffentlichkeit miteinander reden könnten. Insgesamt aber blieb diese Lösung eher vage. ${ }^{13}$

Völlig unterschätzt aber hatten die Abgeordneten die Erwartungshaltung, die ihr Wahlkampf in der (medialen) Öffentlichkeit, aber auch in der eigenen Partei geschaffen hatte. Nach den ersten Reaktionen auf die Debatte beschwichtigten sie schon am nächsten Tag: Sie stellten klar, dass alle Fraktionssitzungen öffentlich sein würden, und von „Zensur“ war keine Rede mehr. ${ }^{14}$ Damit war die Kontroverse zwar zunächst vom Tisch, das eigentliche Problem aber nicht gelöst. Das wurde bei der konstituierenden Sitzung der neuen Fraktion drei Tage später deutlich. Im Vorfeld hatten sich der Spitzenkandidat Andreas Baum und der medial präsenteste Abgeordnete Christopher Lauer abgesprochen, gemeinsam als Doppelspitze für den Fraktionsvorsitz zu kandidieren. Susanne Graf und Gerwald Claus-Brunner kritisierten das als genau die Art von Politik, gegen die sich die Partei zu Recht wende: Absprachen in Hinterzimmern, bei denen für die Wähler nicht transparent werde, wer warum wofür votiert habe. Da der Streit (sowohl um die Transparenz als auch um die Posten) kein gutes Bild der Partei in der Öffentlichkeit abgäbe, forderte Lauer im Gegenzug, bestimmte sensible Entscheidungen nichtöffentlich zu treffen, um mediale Schlammschlachten zu vermeiden. ${ }^{15}$ In der darauffolgenden Woche wurde schließlich Baum in einer geheimen Abstimmung zum alleinigen Fraktionsvorsitzenden gewählt. ${ }^{16}$ Somit wurde zwar dieser konkrete Streit beendet, der grundsätzliche Konflikt aber blieb ungelöst und konnte immer wieder virulent werden. Auf der einen Seite standen dann die Abgeordneten, die für einen pragmatischen Umgang mit dem Thema „Transparenz“ eintraten und vor allem Streitigkei-

13 Vgl. ebenda; außerdem Karin Christmann, Piraten streiten über Transparenz, in: Der Tagesspiegel vom 21. September 2012, S. 8.

14 Vgl. Svenja Bergt / Benjamin Quiring, Piraten üben Demokratie. Das Kreuz mit der Transparenz, in: taz vom 22. September 2011, S. 22.

15 Vgl. Piratenfraktion Berlin, Erste Sitzung der Fraktion am 22. September 2011, http://www.piratenfraktion-berlin.de/wp-content/uploads/2011/10/FraSitz2011-09-22.pdf (Abruf am 6. April 2012); Johannes Schneider, Streit um sechs, in: Der Tagesspiegel vom 23. September 2011, S. 7.

16 Vgl. Christina Brüning, Habemus Satzung, Habemus Captain, in: Berliner Morgenpost vom 28. September 2011, S. 20. 
ten sowie längerfristige strategische Planungen, die Verteilung von Ämtern, aber auch beispielsweise unausgereifte inhaltliche Vorschläge von der Medienöffentlichkeit fern halten wollten. Auf der anderen Seite fanden sich die Abgeordneten, die für das gegebene Versprechen, Transparenz nur zum Schutz von personenbezogenen Daten ausnahmsweise aufzuheben, eintraten und forderten, die Piratenpartei dürfe hier eben nicht wegen vermeintlicher Sachzwänge wie die anderen Parteien im Parlament werden. Interessant ist hierbei auch, dass beide Gruppen mit der Außenwirkung und der zukünftigen Wählbarkeit argumentierten: Für die einen ist der permanente Streit in der Öffentlichkeit, für die anderen das Aufgeben der Parteiideale gefährlicher für das Ansehen der Partei.

In vielen Punkten entschied sich die Fraktion zunächst für beispiellose Transparenz, die sich so in keiner anderen Parlamentsfraktion finden lässt. Die Sitzungen der Piratenfraktion und alle sie betreffenden Anträge sind bis heute in der Regel öffentlich zugänglich, das Protokoll wird während der Sitzung live online erstellt und kann kommentiert werden; außerdem gibt es einen Livestream, man kann also der Fraktionssitzung über das Internet von überall folgen. Lediglich zum Schutz der Persönlichkeitsrechte beispielsweise von Stellenbewerbern wird - wie auch im Wahlprogramm gefordert - die Öffentlichkeit ausgeschlossen. Die Transparenz beschränkt sich aber keineswegs auf die Fraktionssitzungen: Die Termine aller Abgeordneten sind über den Fraktionskalender online einsehbar, ihre Nebeneinkünfte legten sie bereits kurz nach ihrem Einzug ins Parlament, im Februar 2012, umfassend offen. ${ }^{17}$ Dabei sind die Angaben der Piraten wesentlich detaillierter als beispielsweise die der Grünen, die entsprechende Informationen ebenfalls über das vorgeschriebene Maß hinaus veröffentlichen. ${ }^{18}$ Der Haushaltsplan der Piratenfraktion kann detailliert online eingesehen werden. ${ }^{19}$ Interviews wurden von den Abgeordneten nicht, wie sonst üblich, gegengelesen und dann freigegeben, sondern durften einfach veröffentlicht werden - das galt selbst dann noch, als der Parlamentarische Geschäftsführer Martin Delius den Aufstieg der Piraten mit dem der NSDAP verglich und ihm schon vor der Publikation des Interviews klar wurde, welche Konsequenzen das haben musste. ${ }^{20}$ Ebenso veröffentlichte die Fraktion mehrfach vertrauliche Dokumente zum Berliner Flughafen BER, von deren öffentlicher Bedeutung sie überzeugt war, wiederum ungeachtet der möglichen negativen Konsequenzen. ${ }^{21}$ Außerdem stellt die Fraktion ein hohes Maß an Transparenz über interne Vorgänge auch dadurch her, dass ihre internen Gespräche und selbst Streitigkeiten häufig über öffentlich einsehbare

17 Vgl. Karin Christmann / Lars von Törne, Finanzielle Transparenz. Piratenfraktion lässt sich in den Geldbeutel gucken, in: Der Tagesspiegel vom 4. Februar 2012, S. 4. Die einzige Ausnahme war hier der Abgeordnete Alexander Morlang, der sich über ein Jahr lang weigerte, der Aufforderung seiner Parteifreunde nachzukommen. Vgl. dazu Karin Christmann, Pirat legt Verdienste teilweise offen, in: Der Tagespiegel vom 11. Dezember 2012, S. 8; Christian Füller, „Wir wollen Deine Unterhosen sehen“. Berliner Pirat Morlang über Einkünfte, in: taz vom 9. Februar 2013, S. 43.

18 Vgl. Bündnis 90/Die Grünen, Beschluss der Fraktion vom 19. Juni 2012. Veröffentlichung von Einkünften der MDA, http://www.gruene-fraktion-berlin.de/sites/default/files/Beschluss.pdf (Abruf am 15. Mai 2014).

19 Vgl. Piratenfraktion Berlin, Beschlossener Haushaltsplan 2013, https://docs.google.com/spreadsheet/ccc?key=0AugSBU1V1hU7dFVzRnpzTTN4ZWtGMXhTc1ZLVXdadWc\#gid=0 (Abruf am 3. Juli 2013).

20 Vgl. Sabine Rennefanz, Delius will die Attacken aushalten, in: Berliner Zeitung vom 24. April 2012, S. 2.

21 Vgl. Lars von Törne, Flughafen-Debakel: Wowereit fühlt sich von Piraten verraten, in: Der Tagesspiegel vom 9. August 2012, S. 10. 
Mailinglisten, Blogs oder Twitter geführt und so von einem interessierten Publikum verfolgt werden können.

Diesen bisher von keiner anderen Fraktion jemals angestrebten hohen Grad an Transparenz zu erreichen, war für die Piraten nicht leicht. Vieles war so noch nie versucht worden; technische und organisatorische Schwierigkeiten wurden dadurch verstärkt, dass sich die Fraktion in sehr vielen Bereichen einarbeiten musste und so die Herstellung von Öffentlichkeit nicht immer den höchsten Stellenwert hatte. Festzuhalten ist jedoch, dass man nach der schwierigen Anfangszeit sichtlich bemüht war, die Transparenz zu erhöhen - und wie dargestellt auch viele Erfolge vorzuweisen hat. Durch den Aufbau der Internetseite der Fraktion $^{22}$, das Einrichten offizieller Twitter-Accounts von einigen Abgeordneten, genauere und korrektere Protokolle und ähnliches mehr wurde das Transparenzversprechen schrittweise weiter umgesetzt. Gleichzeitig aber fanden die zu Beginn von der Mehrheit der Fraktion noch zurückgewiesenen Argumente, ohne Öffentlichkeit zielführender arbeiten zu können und das Bild in der Öffentlichkeit stärker selber zu bestimmen, unter den Abgeordneten mehr und mehr Befürworter. Besonders hinsichtlich der öffentlich zugänglichen Fraktionssitzungen ist das deutlich zu beobachten.

Zunächst waren Ausnahmen von der Regel, grundsätzlich öffentlich zu tagen, besondere Ereignisse, für die die Fraktion sich rechtfertigte und teilweise im Nachhinein entschuldigte. Als sie sich beispielsweise kurz nach der Wahl ohne Kameras und ohne Protokoll nach Tschechien in Klausur begab, stellte sie hinterher klar, man habe sich hauptsächlich kennengelernt, über Politik sei nicht gesprochen worden. ${ }^{23}$ Nachdem Ende Februar 2012 die Öffentlichkeit für die Sitzung ausgeschlossen worden war, in der die Fraktion zwei Mitglieder für die Bundesversammlung wählen durfte, protestierte die Parteibasis - und der damalige Parlamentarische Geschäftsführer Martin Delius räumte ein, dass dies ein Fehler gewesen sei. ${ }^{24}$ Im Juni 2012 stellte sich das Bild dann schon etwas anders dar: Die Fraktion zog sich für fünf Tage nach Potsdam zurück, um grundlegende Fragen zur politischen Arbeit und zur Strategie zu besprechen. Auch über die Neuordnung an der Fraktionsspitze und über die Verteilung der Ausschüsse wurde diskutiert. Im Gegensatz zur ersten Klausur in Tschechien stritten die Abgeordneten diesmal auch nicht ab, dass es hauptsächlich um politische Inhalte gehen sollte. Die damals amtierende Vorsitzende des Landesverbandes der Piratenpartei, Christiane Schinkel, erklärte lediglich, „der Transparenz sei Genüge getan, wenn im Anschluss an die Tagung über die Ergebnisse berichtet werde“25. Die Fraktion erklärte auf ihrer Internetseite, in einer öffentlichen Sitzung hätten die Abgeordneten nicht

22 Auch nach zwei Jahren funktioniert die Internetseite der Fraktion nicht wie von dieser selbst angekündigt und gewünscht. Dadurch können einige Vorhaben für mehr Transparenz immer noch nicht umgesetzt werden. Beispielsweise ist der Fraktionskalender seit Spätsommer 2013 aufgrund technischer Schwierigkeiten nicht erreichbar, die angekündigte Ersatzlösung aber im Mai 2014 immer noch nicht gefunden.

23 Vgl. Karin Christmann / Claudia Maier, Demnächst heißt es: liefern, in: Der Tagesspiegel vom 13. Oktober 2011, S. 11.

24 Vgl. Martin Delius, Martin Delius zu Nominierungen zur Bundesversammlung, eigentliche Nachricht vom 28. Februar 2012, http://www.piratenfraktion-berlin.de/2012/02/29/martin-delius-zu-nominierungen-zur-bundesversammlung/ (Abruf am 8. März 2012).

25 Karin Christmann, Großes Piratengeheimnis, in: Der Tagesspiegel vom 19. Juni 2012, S. 7; vgl. auch Christina Brüning, Piraten wollen eine Doppelspitze einführen, in: Berliner Morgenpost vom 22. Juni 2012, S. 10. 
so frei sprechen können, dazu sei ein „vertrauliches Umfeld“26 notwendig. Während also im Herbst 2011 die politische Bedeutung der ersten Klausur noch heruntergespielt wurde, begründeten die Piraten nun offensiv die Notwendigkeit nichtöffentlicher Räume - auch wenn es innerhalb der Fraktion harsche Kritik an diesem „Mangel an Transparenz" gab.

Die Fraktionssitzung, in der dann der neue Fraktionsvorstand gewählt wurde, war zwar wie gewohnt öffentlich und wurde im Netz übertragen; es wurde aber deutlich, dass die relevanten Entscheidungen schon im Vorfeld abgesprochen worden waren. Nach dem überraschenden Rückzug mehrerer Kandidaten waren lediglich noch Andreas Baum und Christopher Lauer übrig, um die Doppelspitze - deren Einführung ebenfalls nicht transparent diskutiert wurde - zu besetzen. Wie schon im Herbst 2011 regte sich innerhalb der Fraktion dagegen Widerstand: Wolfram Prieß beispielsweise bezeichnete die Wahl als „Farce“ und „abgekartetes Spiel“27. Dieses Mal konnten sich die Kritiker allerdings nicht durchsetzen Baum und Lauer wurden gewählt.

Noch weiter entfernt von ihrem ursprünglichen Ideal zeigte sich die Fraktion im Vorfeld der Neuwahl der Fraktionsspitze im Mai 2013. Am Freitagabend vor Pfingsten berief Lauer eine Pressekonferenz ein, auf der er ausführte, warum gegen ihn gestreute Verleumdungen - es ging um seine Beziehung zu einer Mitarbeiterin sowie um die Einstellung und Beförderung von deren Mutter - haltlos seien. Vielmehr würden die Gerüchte in die Welt gesetzt, um ihn im Vorfeld der Neuwahl des Fraktionsvorstandes zu beschädigen. ${ }^{28}$ Der Urheber müsse einer der Abgeordneten sein, der in der nächsten Fraktionssitzung ermittelt und bestraft werden solle. Die Öffentlichkeit sei zu dieser Aussprache nicht zugelassen, da es kein Recht darauf gäbe, den Streit, obgleich um ein politisches Amt, mitzubekommen. ${ }^{29}$ Die entsetzten Reaktionen zahlreicher Abgeordneter ${ }^{30}$ bezogen sich nicht nur auf die Tatsache, dass sie über die Vorgänge selbst aus der Presse erfahren mussten, sondern auch auf den angekündigten Ausschluss der Öffentlichkeit. Fabio Reinhardt beispielsweise forderte eine transparente Aufklärung und sah für die Fraktion die „Verpflichtung, die Debatte soweit nicht zwingend anders erforderlich - öffentlich zu führen "31. Während in der Fraktionssitzung am Dienstag nach Pfingsten einige Abgeordnete versuchten, wenigstens die

26 Piratenfraktion Berlin, Fraktionsklausur in Potsdam und Fraktionssitzung am 22. Juni 2012, https://www.piratenfraktion-berlin.de/2012/06/21/fraktionsklausur-in-potsdam-und-fraktionssitzung-am-22-juni-2012/ (Abruf am 3. Juli 2013).

27 Sabine Beikler, Kabale und Piratenliebe, in: Der Tagesspiegel vom 23. Juni 2012, S. 18. Vgl. auch Piratenfraktion Berlin, Außerordentliche Fraktionssitzung am 22. Juni 2012, https://www.piratenfraktion-berlin.de/wp-content/uploads/2011/10/ausserordentliche-FraSi_2012_06_2012.pdf (Abruf am 10. Juli 2013).

28 Dass Lauer, Baum und Herberg auf dieser Sitzung ihre intern getroffene Absprache, in dieser Konstellation erneut für den Vorstand kandidieren zu wollen, bekannt gaben, sorgte für Kritik, da hier die Entscheidungsprozesse nicht transparent dargestellt wurden. Baum und Lauer zogen ihre Kandidaturen später zurück.

29 Vgl. Karin Christmann, Piraten suchen das U-Boot, in: Der Tagesspiegel vom 18. Mai 2013, S. 14; Piratenfraktion Berlin, Audiomitschnitt der Pressekonferenz vom 17. Mai 2013, https:// www.piratenfraktion-berlin.de/2013/05/17/pressekonferenz-vom-17-5-2013/ (Abruf am 9. Juli 2013).

30 Vgl. beispielsweise Susanne Graf, Ich möchte kein Spielball sein, 17. Mai 2013, http://www.grafsusanne.de/wordpress/ich-mochte-kein-spielball-sein/ (Abruf am 10. Juli 2013).

31 Fabio Reinhardt, Meine Fragen an den Fraktionsvorstand, 21. Mai 2013, http://pirat.fabioreinhardt.de/kommentar/meine-fragen-an-den-fraktionsvorstand/ (Abruf am 10. Juli 2013). 
nicht personenbezogenen Fragen im öffentlichen Sitzungsteil zu besprechen, votierte eine knappe Mehrheit von sieben zu sechs Stimmen bereits kurz nach Beginn der Diskussion für einen Ausschluss der Öffentlichkeit. ${ }^{32}$ Auch in der Sitzung in der darauffolgenden Woche wurde das Thema noch einmal nichtöffentlich diskutiert ${ }^{33}$ und anschließend eine Erklärung veröffentlicht, in der die Fraktion ihre Gemeinsamkeiten betonte und für die Zukunft ein besser abgestimmtes Vorgehen und eine bessere interne Zusammenarbeit versprach. ${ }^{34}$ Die vielen Unklarheiten im Zusammenhang mit den Vorfällen der vorangegangenen Tage wurden nicht thematisiert, um den Streit nicht neu zu entfachen. Antworten auf die größtenteils politisch relevanten Fragen erhielt die Öffentlichkeit also nicht.

Dabei ist rein quantitativ keine generelle Tendenz feststellbar: Der Anteil nichtöffentlicher Fraktionssitzungen hat sich in den ersten zwei Jahren von einem niedrigen Ausgangswert nicht deutlich erhöht; die Zahlen schwanken zwar, ein Trend ist aber nicht zu erkennen. Interessanter als die reinen Zahlen sind der Umgang mit der Thematik und die Argumente in der Auseinandersetzung. Die geschilderte Abfolge der Ereignisse zeigt dabei den permanenten Konflikt innerhalb der Fraktion sowie dessen Gründe: War die Fraktion idealistisch, aber ohne genaue Vorstellungen der Arbeit im Abgeordnetenhaus gestartet, gewannen pragmatische Erwägungen insbesondere zu Außendarstellung und Arbeitsfähigkeit langsam die Oberhand. Beim Thema „Öffentliche Fraktionssitzungen“ hat diese Entwicklung sicher die größte Bedeutung (und die meiste Medienaufmerksamkeit), sie lässt sich aber auch an anderen Punkten ablesen. Mussten, wie erläutert, zu Beginn Interviews überhaupt nicht autorisiert werden (und war manchmal sogar das komplette, unbearbeitete Gespräch abrufbar ${ }^{35}$ ), wurden einige Abgeordnete im Laufe der Zeit deutlich rigoroser, was die Freigabe von Zitaten und Aufnahmen durch Journalisten angeht. ${ }^{36}$ Andere Abgeordnete hingegen behielten ihren offenen Umgang mit Pressevertretern bei. Mithilfe der Pressestelle versuchte daher vor allem die Fraktionsführung, die professionelleren, aber von Pressevertretern als streng empfundenen Regeln für alle Abgeordneten durchzusetzen. So sollte beispielsweise zukünftig ein Mitarbeiter der Pressestelle bei allen Interviews zugegen sein und diese aufzeichnen. Auch die Regeln für die Autorisierung von Zitaten sollten verschärft werden. Die in den erwähnten Beschlüssen zur Arbeit innerhalb der Fraktion von Mai 2013 zu findende Aufforderung, eine „gemeinsame Presse- und Öffentlichkeitsarbeit über die Pressestelle ohne Alleingänge" 37 zu machen, wurde denn auch nicht von allen lediglich als Richtlinie zu einer besseren Koordination mit der Pressestelle gesehen. ${ }^{38}$ Erst ein Beschluss der Fraktion auf Antrag ihres neuen Vorsitzenden Oliver Höfinghoff stellte klar, dass

32 Vgl. Piratenfraktion Berlin, 68. Fraktionssitzung am 21. Mai 2013, https://www.piratenfraktionberlin.de/wp-content/uploads/2011/10/2013_05_21_FraSi_KORR.pdf (Abruf am 10. Juli 2013).

33 Vgl. dies., 69. Fraktionssitzung am 28. Mai 2013, https://www.piratenfraktion-berlin.de/wpcontent/uploads/2011/10/2013_05_28_FraSi.pdf (Abruf am 10. Juli 2013).

34 Vgl. dies., Beschlüsse der Fraktionssitzung vom 28. Mai 2013, https://www.piratenfraktion-berlin.de/2013/05/29/beschlusse-der-fraktionssitzung-vom-28-mai-2013/ (Abruf am 10. Juli 2013).

35 So das wenige Wochen vor der Wahl von der taz geführte Interview mit Andreas Baum: Gereon Asmuth / Svenja Bergt, „Mir ist egal, neben wem ich sitze“, in: taz.de vom 7. September 2011, http://www.taz.de/!77664/ (Abruf am 28. Juli 2013).

36 Vgl. u.a. Nina Pauer / Khue Pham / Heinrich Wefing, Die große Vergiftung, in: Die Zeit vom 16. Mai 2013, S. 2 f.; Astrid Geisler, Die Verwandlung, in: taz vom 11. Juni 2013, S. 5.

37 Piratenfraktion Berlin, a.a.O. (Fn. 34).

38 Vgl. Karin Christmann, Der Neue twittert kaum, in: Der Tagesspiegel vom 12. Juni 2013, S. 10. 
die Pressestelle nicht die Aufgabe habe, „Interviews von Fraktionsmitgliedern elektronisch aufzuzeichnen und zu speichern“ und nur dann bei Interviews dabei sein dürfe, „falls dies von den Abgeordneten gewünscht wird“" ${ }^{39}$. In diesem Punkt wurde also dem Versuch, eine Abkehr vom Transparenzgedanken festzuschreiben, von der Mehrheit der Fraktion mit einiger Verzögerung explizit eine Absage erteilt. Die einzelnen Abgeordneten können mit dem Thema aber natürlich weiterhin sehr unterschiedlich umgehen.

Ein weiterer Punkt, bei dem die ursprünglich praktizierte Transparenz reduziert wurde, ist der Fraktionsvorstand. Tagte er zunächst öffentlich und fanden sich seine Protokolle im Netz, wurden diese Treffen Ende 2011 aus Effizienzgründen eingestellt. Die weiterhin stattfindenden informellen Treffen waren für die Öffentlichkeit nicht mehr nachvollziehbar, und die im Herbst 2012 unter dem neuen Vorstand wieder eingeführten Sitzungen waren sogar für die restlichen Abgeordneten unzugänglich (mehr dazu in Abschnitt 1.3.).

Das größte Problem für die Umsetzung der versprochenen Transparenz in der Fraktion aber ist die zunehmende Verlagerung politisch relevanter Debatten aus den öffentlich einsehbaren Institutionen und Medien. Abseits der zuständigen Gremien werden Entscheidungen von Gruppen von Abgeordneten vorbesprochen und miteinander abgestimmt, so dass später in der öffentlichen Debatte die Entstehung eines Antrages und die Gründe für seine Befürwortung nicht mehr klar ersichtlich werden. Auch wenn diese Ausweichbewegungen von einigen Abgeordneten kritisiert werden, lassen sie sich natürlich nicht einfach verbieten. Bisher hat die Fraktion hier keine Antwort gefunden, beispielsweise durch die Veröffentlichung der Entstehungsgeschichte eines jeden Antrages.

Als Fazit lässt sich festhalten, dass die Fraktion der Berliner Piratenpartei mit der $-\mathrm{zu}$ Beginn noch etwas holprigen - Umsetzung ihres Transparenzversprechens in der Tat etwas qualitativ völlig Neues ins Parlament gebracht hat. Die dadurch erzeugte Aufmerksamkeit führte dazu, dass auch alle anderen Parteien das Thema fortan auf die Agenda setzten. In einigen wichtigen Punkten hat die Fraktion diese Transparenz zwar inzwischen zurückgefahren und sich insoweit den Erfordernissen des politischen Betriebes angepasst; zieht man aber die anderen Fraktionen des Abgeordnetenhauses als Vergleichsmaßstab heran, arbeiten die Piraten insgesamt immer noch sehr transparent.

\subsection{Basisbeteiligung}

Die in den Medien als Mitmachpartei bezeichneten Piraten plakatierten im Berliner Wahlkampf unter anderem den Slogan: „Wir sind die mit den Fragen, Ihr seid die mit den Antworten. Wir PIRATEN entwickeln neue Formen der Bürgerbeteiligung und der Demokratie und setzen sie um. " ${ }^{40}$ Sie gingen mit der Überzeugung ins Parlament, der einzelne Abgeordnete sei letztlich zur Umsetzung basisdemokratisch getroffener Beschlüsse verpflichtet, wobei sie sich dem Konflikt dieser Auffassung mit dem freien Mandat durchaus bewusst waren.

39 Piratenfraktion Berlin, Richtlinien für die Fraktion zur Pressearbeit, Antrag vom 21. Juni 2013, https://redmine.piratenfraktion-berlin.de/issues/7025 (Abruf am 30. Juli 2013).

40 Das Plakat kann eingesehen werden unter http://wiki.piratenpartei.de/Datei:Piraten-AGH-WahlFragen-und-Antworten.jpg (Abruf am 2. August 2013). 
Um dem Anspruch, den Willen der Parteibasis umzusetzen, gerecht zu werden, nutzte die Fraktion von Anfang an das zumindest im Berliner Landesverband der Piratenpartei bereits etablierte „LiquidFeedback“ (LQFB). Mit dieser Software können theoretisch alle Berliner Piraten über Initiativen abstimmen, Verbesserungsvorschläge machen oder selbst Initiativen einbringen, die dann von den anderen Teilnehmern abgestimmt werden. Dabei verfügt jedes Mitglied über eine Stimme, die es entweder selbst vergeben oder an ein anderes Mitglied delegieren kann. Dieses Mitglied kann dann entweder selbst mit zwei Stimmen abstimmen, oder es delegiert die zwei Stimmen wiederum an ein anderes Mitglied, wobei jede Stimmendelegation vor dem Ende der jeweiligen Abstimmung rückgängig gemacht werden kann. ${ }^{41}$ Allerdings ist das LiquidFeedback in der Piratenpartei nicht unumstritten: Kritiker bemängeln unter anderem die durch die Stimmendelegation entstehenden „Superdelegierten“, organisatorische Probleme (während manche Mitglieder zwei Accounts besitzen, warten andere monatelang auf ihren) sowie die fehlende Nachvollziehbarkeit der Ergebnisse da nicht mit Klarnamen abgestimmt werden muss, könne niemand ausschließen, dass das Abstimmungsergebnis manipuliert sei. ${ }^{42}$

Nach ihrem Einzug ins Abgeordnetenhaus nahmen sich die Piraten vor, alle politischen Entscheidungen, die im Plenum des Parlaments zu treffen sind, vorher ins LQFB zu stellen und so das Votum der Basis einzuholen. Dabei sollte es nicht nur um die eigenen Anträge gehen, sondern auch um die der anderen Fraktionen. Nur Entscheidungen, die die Organisation der Fraktion betreffen, sollten davon ausgenommen sein. ${ }^{43}$

In der ersten Zeit nach der Wahl bemühte sich die neue Fraktion, ihre eigenen Ansprüche umzusetzen. In ihrer ersten Satzung formulierte sie, die Abgeordneten sollten neben dem Wahlprogramm, der Mehrheitsmeinung der Fraktion und Parteitagsbeschlüssen des Berliner Landesverbandes auch Entscheidungen des LiquidFeedbacks „berücksichtigen“44. Mitarbeiter der Fraktion begannen, die anstehenden Entscheidungen ins LQFB einzustellen. Die Basis nahm das Angebot teilweise euphorisch an und stellte zu den verschiedensten Themen eigene Anträge, die jedoch oft nicht ausgereift oder ausformuliert waren. Allerdings beteiligten sich schon in der Anfangszeit oft weniger als einhundert Mitglieder an Abstimmungen, und selbst bei kontroversen oder für die Partei wichtigen Themen waren es so gut wie nie mehr als zehn Prozent der stimmberechtigten Basis - trotz der vergleichsweise geringen Opportunitätskosten bei Abstimmungen über das Internet. ${ }^{45}$ Hinzu kommt, dass aufgrund der möglichen Stimmendelegation die Anzahl derer, die sich tatsächlich beteiligt haben, noch deutlich niedriger liegt.

41 Vgl. Sebastian Buck, Liquid Democracy - eine Realisierung deliberativer Hoffnungen? Zum Selbstverständnis der Piratenpartei, in: ZParl, 43. Jg. (2012), H. 3, S. 626 - 635, S. 626 f.

42 Vgl. Alexander Hensel / Stephan Klecha, Zwischen Stammtisch und Etherpad. Beteiligung und Mitwirkung in der Piratenpartei, in: Forschungsjournal Soziale Bewegungen, 26. Jg. (2013), H. 2, S. $62-71$, S. $65 \mathrm{ff}$.

43 Vgl. Carsten Koschmieder, a.a.O. (Fn. 3), S. 216 f.

44 Piratenfraktion Berlin, Satzung, $\$ 13$, https://www.piratenfraktion-berlin.de/wp-content/uploads/2011/10/satzung.pdf (Abruf am 12. August 2013).

45 Die Ergebnisse der Abstimmungen finden sich unter https://qpp.de/be/unit/show/1.html (Abruf am 12. August 2013). Die Bemühungen der Fraktion, auf ihrer Webseite zu dokumentieren, inwiefern erfolgreiche LQFB-Initiativen der Basis aufgegriffen wurden und was im Parlament aus ihnen geworden ist, kommen auch nach zwei Jahren nicht über ein Anfangsstadium hinaus. Vgl. dazu Piratenfraktion Berlin, Anträge, https://www.piratenfraktion-berlin.de/antrage-piratenfraktion/liquid-feedback/ (Abruf am 17. September 2013). 
In der Praxis der parlamentarischen Arbeit zeigte sich schnell, dass die Vorstellungen zum Umgang mit LQFB nicht umsetzbar waren. Die Kapazitäten der Fraktion und ihrer Mitarbeiter, die durch ihren Neuaufbau an vielen Stellen überlastet waren, reichten nicht aus, um alle Anträge immer sofort zu bearbeiten. Viele Entscheidungen müssen kurzfristig getroffen werden, eine Abstimmung im LQFB benötigt aber in der Regel mehr als eine Woche, um zu einem sinnvollen Ergebnis zu kommen. Aufgrund dieser Schwierigkeiten änderte die Fraktion nach und nach ihre Ansprüche. Heute werden vor allem die Anträge der eigenen Mitglieder im LQFB abgestimmt, die anderer Fraktionen oder auch gemeinsame Anträge, die die Piraten mit anderen Fraktionen zusammen einbringen, hingegen nicht. Auch Anträge, die Inhalte aus dem Wahl- oder dem Parteiprogramm aufnehmen, werden der Basis oft nicht mehr vorgelegt. Wiederum gibt es dabei große Unterschiede zwischen einzelnen Abgeordneten: Während einige versuchen, bei jedem eigenen Antrag vorher die Basis zu befragen, nehmen andere das nicht sonderlich wichtig.

Neben den organisatorischen Schwierigkeiten gibt es zwei weitere wichtige Gründe, warum die Piratenfraktion sich von ihrer ursprünglichen Idee der radikalen Basisbeteiligung verabschiedet hat: die geringe Beteiligung an den Partizipationsangeboten einerseits sowie die Professionalisierung der Fraktionsmitglieder andererseits. ${ }^{46} \mathrm{Zu}$ Beginn der Wahlperiode waren die Abgeordneten neu in ihrer Rolle als Berufspolitiker und hatten keine Erfahrung mit der Arbeit im Parlament. Die Basis war nach dem überraschenden Wahlerfolg gespannt auf die neuen Möglichkeiten der Mitbestimmung, die es so noch nicht gegeben hatte, und probierte sie aus. Im Laufe der ersten zwei Jahre dann ging die anfängliche Begeisterung der Basis aber zurück, vermutlich einerseits, weil der Reiz des Neuen verflog, andererseits aber auch aufgrund der Tatsache, dass die Anträge einer kleinen Oppositionsfraktion naturgemäß oft wirkungslos blieben. So nahm die Beteiligung am LQFB ab, obwohl sich die Mitgliederzahl in Berlin seit der erfolgreichen Wahl 2011 verdreifacht hat. ${ }^{47}$ Für die Abgeordneten aber hat das Ergebnis einer Abstimmung, an der von den offiziell über 3.000 Parteimitgliedern nur drei Dutzend teilgenommen haben, kaum Verbindlichkeit.

Neben der sinkenden Beteiligung sorgte auch die Professionalisierung für einen veränderten Umgang mit dem Input der Basis. Im Laufe der Zeit arbeitete sich die Fraktion in den Parlamentsbetrieb ein und gewann durch die permanente Beschäftigung mit Inhalten und Prozessen sowie durch die Zuarbeit der Referenten einen stetig wachsenden Wissensvorsprung gegenüber den Parteimitgliedern, die politikfernen Beschäftigungen nachgehen. Das Bereitstellen, Aufarbeiten und Zusammenfassen notwendiger Informationen für die einfachen Mitglieder gelingt den Abgeordneten (noch) nicht ausreichend. Der Hauptgrund hierfür ist wiederum vor allem das Fehlen der enormen zeitlichen und personellen Ressourcen, die dafür notwendig wären; bei einigen Abgeordneten ist aber auch ein Unwille zu erkennen, die Parteimitglieder stärker einzubinden. Zwar gab es auch an der Basis einen permanenten Lernprozess, und viele Anträge sind heute ausgereifter und strukturierter als

46 Hinzu kommt, dass die Fraktion die Kritik an der mangelnden Nachvollziehbarkeit der Ergebnisse im LQFB teilt und stattdessen ein Abstimmungssystem bevorzugen würde, in dem alle Teilnehmer mit ihrem Klarnamen abstimmen müssen. Diese Abstimmungen würden dann stärker in die Arbeit der Fraktion einfließen. Siehe dazu Piratenfraktion Berlin, Stellungnahme der Fraktion zum LQFB, Antrag vom 27. November 2012, https://redmine.piratenfraktion-berlin.de/issues/4692 (Abruf am 11. August 2013).

47 Die Mitgliederentwicklung findet sich unter http://wiki.piratenpartei.de/BE:Mitglieder (Abruf am 10. August 2013). 
zu Beginn; durch die Fluktuation unter den Aktiven geht solch erlerntes Wissen aber teilweise wieder verloren. Ausgleichen können die Mitglieder diese Nachteile im Prinzip nur, wenn sie bereits in der Vorbereitungsphase des Antrages mit dem entsprechenden Fachabgeordneten oder seinen Referenten zusammenarbeiten. Diese Praxis allerdings passt kaum noch zu der anfänglichen Idee, die Fraktion sei für die Umsetzung des Basiswillens zuständig und solle keinerlei Führungsfunktion übernehmen. Stattdessen sehen viele Abgeordnete durch den offensichtlichen Wissensvorsprung immer weniger Sinn in der ursprünglich intendierten Art der Beteiligung. ${ }^{48}$ So werden heute auch im LQFB beschlossene Anträge von den Abgeordneten gegebenenfalls nochmals verändert, und nicht alle Parlamentarier sehen die Notwendigkeit, die geänderten Anträge erneut ins LQFB zu stellen.

Zusammenfassend lässt sich sagen, dass sich der Ansatz der Basiseinbindung sowie die Umsetzung mittels LiquidFeedback tatsächlich von den anderen Fraktionen unterscheiden. Mittlerweile hat aber sowohl die Beteiligung der Basis als auch der Wille der Abgeordneten, die Basis einzubeziehen, stark nachgelassen. Von der ursprünglichen Idee ist wenig geblieben.

\subsection{Hierarchie und Fraktionsdisziplin}

Die Politische Kultur in der Piratenpartei sieht institutionalisierte Macht sehr kritisch, gerade wenn sie auf wenige Schultern verteilt ist. Die Vorstände der Partei haben dementsprechend wenig Einfluss auf die Richtung der Partei, sprechen selten auf Parteitagen, werden von der Basis kritisch beobachtet und häufig respektlos behandelt. Die neu gebildete Fraktion hat sich aufgrund dieser herrschaftskritischen Tradition zunächst für eine flache Hierarchie und einen nicht mit den üblichen Machtinstrumenten ausgestatteten Vorsitzenden entschieden. ${ }^{49}$ Damit unterscheiden die Piraten sich deutlich von allen anderen aktuell im Abgeordnetenhaus vertretenen Parteien und werden (nicht nur) in den Medien gern mit den Grünen der frühen achtziger Jahre verglichen. ${ }^{50}$ Übersehen haben die Piraten dabei allerdings, dass die Praxis in den anderen Fraktionen nicht dem Machthunger einzelner Funktionäre, sondern den Funktionslogiken des modernen Parlamentarismus entspringt.

In der zweiten Fraktionssitzung am 27. September 2011 wurden Andreas Baum zum Fraktionsvorsitzenden, Martin Delius zum Parlamentarischen Geschäftsführer sowie Heiko

48 Beispielsweise ermahnte Martin Delius die Basis auf der Landesmitgliederversammlung am 15. September 2012, sich stärker im LiquidFeedback zu beteiligen und Anträge zu stellen, dabei aber auf Qualität zu achten und am besten vorher mit den Fachreferenten zu sprechen. Schlechte Anträge, so stellte er klar, würde er nicht umsetzen.

49 Andere, noch radikalere Vorschläge, die beispielsweise eine Rotation aller notwendigerweise zu besetzenden Posten vorsahen, wurden aber von der Mehrheit abgelehnt.

50 Vgl. u. a. Gregor Boldt, Warum die Piraten die neuen Grünen sind, in: WAZ vom 18. September 2011, http://www.derwesten.de/nachrichten/warum-die-piraten-die-neuen-gruenen-sind-id5075 417.html (Abruf am 28. Juli 2013); Michael Schlieben, Die neuen Grünen, in: Die Zeit vom 4. April 2012, http://www.zeit.de/politik/deutschland/2012-04/piraten-gruene-vergleich (Abruf am 28. Juli 2013); Roman Eichinger / Angelika Hellemann, Sind die Piraten die neuen Grünen?, in: Bild.de vom 22. April 2012, http://www.bild.de/politik/inland/piratenpartei/sind-die-piratendie-neuen-gruenen-teil-i-23786388.bild.html (Abruf am 28. Juli 2013); aber auch Michael Lühmann, Piratenpartei - die Grünen 2.0? Nachfragen zu einem Politikmodell und seiner Übertragbarkeit, in: Kommune, 30. Jg. (2012), H. 1, S. $14-17$. 
Herberg, Fabio Reinhardt und Susanne Graf zu stellvertretenden Vorsitzenden gewählt. Ein klares Indiz für das Fehlen einer Hierarchie war hierbei vor allem, dass von den fünfzehn Abgeordneten nur drei nicht für wenigstens eines der Ämter kandidiert hatten. ${ }^{51}$ Der Fraktionsvorstand bestand somit aus einem Drittel der Fraktion, war also im Vergleich zu den anderen Fraktionen im Berliner Parlament sehr groß. In den ersten Monaten tagte er öffentlich, Gäste waren zugelassen, und es gab einen Livestream; vor allem einfache Abgeordnete besuchten die Sitzungen regelmäßig und meldeten sich auch zu Wort. ${ }^{52}$ Aus diesen Gründen war eine Hierarchie innerhalb der Fraktion zu Beginn kaum vorhanden, und auch die Rolle des Vorsitzenden blieb schwach. Während Lauer, obwohl medial am präsentesten, einfacher Abgeordneter blieb, trat Baum in der Öffentlichkeit kaum in Erscheinung. Dem Politikverständnis der Piraten entsprechend und im Gegensatz zu allen anderen Fraktionen wirkte er fast ausschließlich nach innen, setzte keine inhaltlichen Akzente und versuchte nicht einmal, öffentlich ein Machtwort zu sprechen, was wohl auch unter den Abgeordneten der Piratenpartei kein Gehör finden würde.

Der fünfköpfige Fraktionsvorstand erwies sich jedoch bald als ineffizient. Obwohl die Sitzungen lang und die Diskussionen ausführlich waren, mussten die Argumente in der folgenden Fraktionssitzung erneut ausgetauscht werden, da der Vorstand keine politischen Entscheidungen treffen sollte. Damit kostete die Erfüllung der Funktion der Selbstorganisation die Piraten deutlich zu viel Zeit, die dann für die Beschäftigung mit den wichtigeren repräsentationsbezogenen und regierungsbezogenen Parlamentsfunktionen fehlte. ${ }^{53}$ Daher wurden die offiziellen Treffen bereits Ende 2011 abgeschafft, das Gremium traf sich noch eine Zeit lang informell und dann überhaupt nicht mehr. Als Delius im Juni 2012 sein Amt als Parlamentarischer Geschäftsführer wieder aufgab, um sich auf die Leitung des Untersuchungsausschusses zum Bau des Berliner Flughafens vorzubereiten, wurde daher die Gelegenheit genutzt, die Struktur an der Fraktionsspitze umzubauen. ${ }^{54}$ Im Rahmen der folgenden Neuwahlen wurde der Fraktionsvorstand auf eine Doppelspitze (Baum und Lauer) und einen Parlamentarischen Geschäftsführer (Herberg) verkleinert, gleichzeitig aber die Amtsdauer des Vorstandes auf ein Jahr reduziert. ${ }^{55}$ Fortan gab es wieder Treffen des Fraktionsvorstandes, bei denen aber keine Gäste zugelassen waren. Sogar andere Abgeordnete wurden ausgeschlossen, wodurch der neue Vorstand eine klare Trennung von der restlichen Fraktion herbeiführte. Obwohl die Führung versicherte, nur verwaltende, aber keine politischen Entscheidungen zu treffen, gab es intern Kritik am Entstehen einer Hierarchie zwischen den drei Vorständen und den einfachen Abgeordneten. Dies war von der Fraktion nie vorgesehen, entwickelte sich aber im Laufe der Zeit immer deutlicher. Neben

51 Vgl. Piratenfraktion Berlin, 2. Sitzung der Fraktion vom 27. September 2011, https://www.piratenfraktion-berlin.de/wp-content/uploads/2011/10/FraSitz2011-09-27.pdf (Abruf am 15. Juli 2013).

52 Die Protokolle mit den Namen der jeweils Anwesenden finden sich unter https://www.piratenfraktion-berlin.de/protokolle/ (Abruf am 31. Juli 2013).

53 Vgl. Werner Patzelt, Parlamente und ihre Funktionen, in: ders. (Hrsg.), Parlamente und ihre Funktionen. Institutionelle Mechanismen und institutionelles Lernen im Vergleich, Opladen 2003, S. 13 - 49, S. 22 ff.

54 Vgl. Karin Christmann, Pirat Delius gibt Geschäftsführer-Amt auf, in: Der Tagesspiegel vom 20. Juni 2012, http://www.tagesspiegel.de/berlin/kuenftig-andere-prioritaeten-pirat-delius-gibtgeschaeftsfuehrer-amt-auf/6777332.html (Abruf am 17. Juli 2014).

55 Vgl. Piratenfraktion Berlin, a.a.O. (Fn. 27). 
der Tatsache, dass in der Fraktion auch verwaltende Aufgaben politisch relevant sein können, war für diese Trennung die Tatsache verantwortlich, dass der Vorstand in den Fraktionssitzungen Anträge einbrachte, deren Entstehung für die restlichen Abgeordneten nicht immer nachvollziehbar war. Außerdem versuchte der Vorstand, die Pressearbeit an sich zu ziehen, um nach außen koordinierter aufzutreten. Auch dadurch stieg natürlich sein Einfluss. Die stärker werdende Hierarchie führte in der Fraktion zu massiven Spannungen, aufgrund derer Lauer - der später zu Protokoll gab, ihm als Fraktionsvorsitzenden fehlten eine klare Hierarchie und Sanktionsmöglichkeiten gegen eigenwillige Abgeordnete $^{56}$ - und Baum bei der Neuwahl des Fraktionsvorstandes im Juni 2013 nicht erneut antraten. Die Wahl von Oliver Höfinghoff und Alexander Spies ${ }^{57}$ kann auch als Versuch gewertet werden, die Trennung zwischen Vorstand und Fraktion wieder abzubauen und an die Stellung des Vorsitzenden in den ersten Monaten nach dem Einzug ins Abgeordnetenhaus anzuknüpfen.

Insgesamt scheiterte der Versuch, die Fraktion frei von Hierarchien zu halten, an den Funktionslogiken des modernen Parlamentarismus. Sowohl die grundsätzliche Organisation des Parlamentes sowie die notwendige Zusammenarbeit mit den anderen Fraktionen, als auch die Arbeitsteilung innerhalb der Fraktion bedingen eine Fraktionsführung. ${ }^{58}$ Die zeitweilig starke Hierarchisierung innerhalb der Piratenfraktion sollte aber nicht zu einer ausschließlich negativen Beurteilung führen. Diese ließe außer Acht, dass die Piraten dieses Problem inzwischen erkannt haben und dagegen vorzugehen versuchen - über den Erfolg lässt sich jetzt noch nicht urteilen.

Unbemerkt hingegen blieben die informellen Hierarchien, die sich aufgrund des Fehlens klassischer offizieller Hierarchien in der Fraktion bildeten. Einflussreicher sind beispielsweise jene Abgeordneten, die aufgrund persönlicher oder politischer Beziehungen in engem Kontakt mit vielen (wichtigen) anderen Parlamentariern stehen, während die sozialen oder politischen Außenseiter der Fraktion gerade in informelle Entscheidungsstrukturen nicht eingebunden werden. Weiterhin können natürlich rhetorisch begabte Abgeordnete oder solche, die wegen bestimmter (oft unpolitischer) Eigenschaften medial präsenter sind, aufgrund ihrer öffentlichen und parteiinternen Reputation Einfluss generieren. Diese informellen Hierarchien sind natürlich nichts Piratenspezifisches; ihnen kommt aber in einer Fraktion, die bewusst die Gleichheit aller Abgeordneten in den Vordergrund stellt und auf formelle Hierarchien verzichten will, besondere Bedeutung zu.

Die schwache Stellung des Fraktionsvorstandes und die Betonung der Gleichheit aller Abgeordneten sowie des freien Mandats führen bei den Piraten direkt zur Ablehnung der Fraktionsdisziplin, die sie als „Fraktionszwang“ ansehen. ${ }^{59}$ So war schon vor der Wahl klar,

56 Das entsprechende Interview mit Christopher Lauer kann nachgehört werden unter http://www. wrint.de/2013/07/19/wr193-im-lauers-buro-ihrer-majestat/ (Abruf am 2. August 2013).

57 Heiko Herberg blieb Parlamentarischer Geschäftsführer. Vgl. Sabine Rennefanz, Stimmen für die Streitschlichter, in: Berliner Zeitung vom 12. Juni 2013, S. 17.

58 Vgl. Werner Patzelt, Der Bundestag, in: Oscar W. Gabriel / Everhard Holtmann, Handbuch Politisches System der Bundesrepublik Deutschland, München 2005, S. 159 - 231, S. 228 ff.; Wolfgang Ismayr, Parteien in Bundestag und Bundesregierung, in: Oscar W. Gabriel / Oskar Niedermayer / Richard Stöss (Hrsg.), Parteiendemokratie in Deutschland, Opladen 1997, S. 384 -406, S. $389 \mathrm{ff}$.

59 In Unkenntnis der Funktionslogik des modernen Parlamentarismus schrieb beispielsweise der damalige Parlamentarische Geschäftsführer Martin Delius, die anderen Parteien hätten „völlig 
dass sich die Fraktion der Piratenpartei in diesem Punkt von allen anderen Fraktionen abheben wollte.

Tatsächlich gibt es in der Piratenfraktion keine Probeabstimmungen oder Anweisungen der Fraktionsführung an einzelne Abgeordnete, wie diese abstimmen sollen. In der konstituierenden Sitzung des Abgeordnetenhauses stimmten die Piraten bunt durcheinander, lehnten teilweise Anträge ihrer Fraktionskollegen ab oder enthielten sich. ${ }^{60}$ Durch die hohe Medienaufmerksamkeit für das Verhalten der neuen Parlamentarier wurden die Piraten schnell bekannt dafür, auf die in der Öffentlichkeit unbeliebte Fraktionsdisziplin zu verzichten. In der Praxis aber stimmten die Abgeordneten häufig einheitlich, einerseits natürlich wegen ihrer gemeinsamen politischen Überzeugung gerade bei Fragen, die die Grundwerte der Partei betrafen, andererseits, weil sie sich in den ersten Monaten in der Regel an das per LiquidFeedback eingeholte Votum der Basis hielten - auch das etwas völlig Neues. Es kann argumentiert werden, dass es sich hier um eine neue Form der Fraktionsdisziplin handelt, bei der die aktive Basis der Partei - und nicht die Mehrheit der Fraktion - das Abstimmungsverhalten einzelner Abgeordneter vorgibt. Dagegen spricht vor allem, dass die Basis aufgrund des freien Mandats wesentlich weniger Sanktionsmöglichkeiten gegen einzelne Abweichler hat. Dafür spricht andererseits, dass Piraten, die sich über die Vorgaben der Basis hinwegsetzen, einem hohen Rechtfertigungsdruck ausgesetzt sind. In jedem Fall führten die Abstimmungen im LiquidFeedback in vielen Fällen zu einem geschlossenen Abstimmungsverhalten der Fraktion. Allerdings hat, wie beschrieben, die Bedeutung von LQFB deutlich nachgelassen.

Auch wenn es eine offizielle Fraktionsdisziplin bei den Piraten immer noch nicht gibt, hat sich in diesem Punkt doch einiges getan. Mit der Zeit wurde Abgeordneten klar, dass es in der Funktionslogik arbeitsteilig arbeitender Parlamente liegt, sich nicht in jedes Thema selbst einzulesen, sondern sich „bei Sachfragen auf das Urteil seiner fachlich spezialisierten Kollegen " ${ }^{" 61}$ zu verlassen. Daher versuchen die einzelnen Abgeordneten heute nicht mehr, sich das Für und Wider jedes Antrages selber zu erarbeiten, sondern folgen in der Regel dem entsprechenden Fachpolitiker. ${ }^{62}$ Wird dessen Position von der Mehrheit der Fraktion getragen, muss ein abweichendes Votum im Plenum gut begründet werden, was wiederum nur mit einer genauen Kenntnis des Antrages möglich ist. Abgeordnete, die sich wiederholt nicht an dieses eingespielte Prozedere halten, werden in der Fraktion politisch isoliert, bekommen keine Unterstützung für eigene Anträge und werden bei der Vergabe von Positionen im Parlament übergangen.

vergessen, dass die Debatte im Plenum eigentlich mal der Mehrheitsfindung gegolten hat" und stattdessen den „Fraktionszwang“ eingeführt. Martin Delius, Die Debatte um die Debattenkultur, 2. April 2012, http://martindelius.de/2012/04/die-debatte-um-die-debattenkultur/ (Abruf am 2. August 2013).

60 Vgl. Svenja Bergt, Plätze zu weit rechts, in: taz vom 28. Oktober 2011, S. 7.

61 Werner Patzelt, Wider das Gerede vom „Fraktionszwang“! Funktionslogische Zusammenhänge, populäre Vermutungen und die Sicht der Abgeordneten, in: ZParl, 29. Jg. (1998), H. 2, S. 323 -347, S. 327.

62 In den bereits erwähnten neuen Verhaltensregeln, die sich die Fraktion gegeben hat, findet sich der Satz „Wir lesen die Anträge vorher oder vertrauen unseren FachpolitikerInnen." Piratenfraktion Berlin, a.a.O. (Fn. 34). Vgl. auch Alexander Hensel/ Stephan Klecha, a.a.O. (Fn. 7), S. 78 ff. 
Die Fraktion der Piratenpartei stimmt also heute insgesamt geschlossener ab als zu Beginn der Wahlperiode. Auch wenn durchaus noch Unterschiede zu den anderen Fraktionen zu erkennen sind - insbesondere bei Anträgen, zu denen weder die Basis noch die Fraktion eine Position erarbeitet hat, ist die Abstimmung „freigegeben“-, gleichen sich die Piraten hier doch sehr deutlich an. Die Entwicklung innerhalb der Fraktion lässt darauf schließen, dass sich dieser Prozess eher noch verstärken wird. Der größere Unterschied bleibt also die vergleichsweise flache Hierarchie, die natürlich Auswirkungen auf die Ausgestaltung der sich entwickelnden Fraktionsdisziplin hat, die aber ihrerseits eine potentielle Bedrohung für die bisher flache Hierarchie darstellt.

\subsection{Auftreten}

Dass Abgeordnete und Funktionäre der Piraten eigentlich gar keine Politiker, sondern „normale Menschen“ seien, ist eine in der Partei verbreitete Vorstellung. Von den Berufspolitikern der etablierten Parteien, die sogenannte „Politik 1.0“ betrieben, wollten sich die neugewählten Parlamentarier auch durch ihren Habitus, ihre Sprache, ihre Kleidung, ihr Auftreten absetzen.

Die Fraktion startete ausgesprochen bunt; über ihren unkonventionellen Kleidungsstil und ihre lustigen T-Shirts wurde in der Presse ausführlich berichtet. ${ }^{63}$ Ihre Ausdrucksweise unterschied sich schon durch ihr geringeres Durchschnittsalter und die Verwendung von Begriffen aus dem Internetjargon. Die anderen Parteien im Abgeordnetenhaus warfen den Piraten aber auch recht schnell vor, dass sie für eine Verrohung der Sprache im Plenum und in den Ausschüssen verantwortlich seien. Diese verteidigten sich mit dem Hinweis, ihre Sprache sei eben verständlich und direkt - was zeigt, dass sie sich des Unterschieds bewusst waren und ihn gezielt kultivierten. ${ }^{64}$

Mit der Zeit aber änderte sich der Auftritt der Piraten. Anzüge, Krawatten und Sakkos sind mittlerweile häufiger zu sehen als zu Beginn, wenn auch immer noch seltener als bei den anderen Fraktionen. Die Ausdrucksweise hat sich vor allem in Interviewsituationen gewandelt: Die meisten Abgeordneten reden heute vorsichtiger mit Journalisten und benutzen in solchen Gesprächen eher Floskeln der „Politikersprache“, um nicht falsch verstanden oder falsch zitiert werden zu können. Im Plenum ist diese Veränderung aber weniger zu bemerken.

Insgesamt lässt sich also eine graduelle Anpassung an die Konventionen des politischen Betriebes konstatieren - auch aus dem Bedürfnis beziehungsweise der Notwendigkeit heraus, sich zu professionalisieren. Die Unterschiede in Kleidung, Sprache und Auftreten sind damit aber keineswegs komplett verschwunden.

63 Vgl. u. a. Piratenpartei startet chaotisch ins Parlament, in: Die Welt vom 22. September 2011, http://www.welt.de/regionales/berlin/article13620397/Piratenpartei-startet-chaotisch-ins-Parlament.html (Abruf am 18. Juli 2013).

64 Vgl. Sabine Beikler / Lars von Törne, Politiker streiten um den richtigen Ton, in: Der Tagesspiegel vom 11. April 2012, S. 7. 


\section{Fazit: trotz vieler Konzessionen immer noch „anders“}

Mit dem erstmaligen Einzug der Piratenpartei in ein deutsches Landesparlament haben sich viele Wähler eine Veränderung der Art, wie Politik gemacht wird, erhofft. Deutlich wichtiger als die konkreten sachpolitischen Forderungen der Partei, etwa zur Urheberrechtsreform, erschien vielen, dass die Piraten transparentere Prozesse versprachen, die Bürger stärker einbinden und mitbestimmen lassen wollten. Ihre Kritik an der Praxis der etablierten Parteien mit ihrem „Fraktionszwang“ versprach gleichberechtigte Abgeordnete, die nicht alles „abnicken“ würden, was vom Fraktionsvorstand entschieden wurde. Die frischgebackenen Parlamentarier der Piratenpartei waren ehrlich bemüht, diese Erwartungen zu erfüllen. Keineswegs kann man der Mehrheit von ihnen vorwerfen, dass sie diese Erwartungen nur aus wahltaktischen Gründen geweckt hatten - sie glaubten an diese Ideale. Das Überspringen der Fünfprozenthürde war nicht nur für politische Beobachter eine Überraschung, auch die Partei selbst hatte damit wenige Monate vor der Wahl nicht gerechnet. Folglich hatte sie verständlicherweise kein fertiges Konzept, wie ihre Ideen umzusetzen sein würden. Den Funktionslogiken des modernen Parlamentarismus standen viele Piraten ohnehin eher skeptisch gegenüber - oder hatten sie schlicht nicht verstanden. So startete die Fraktion zwar - wenn auch zunächst etwas holprig - enthusiastisch mit der Umsetzung zahlreicher Neuerungen, musste aber im Laufe der Zeit von einigen wieder abrücken.

Die Abläufe im Abgeordnetenhaus haben die Piraten zweifelsohne transparenter gemacht. Allein die öffentliche Aufmerksamkeit führte dazu, dass sich alle Parteien dem Thema Transparenz zuwandten und Veränderungen vornahmen. Die Piratenfraktion veröffentlicht eine große Zahl von Informationen über ihre Arbeit, die bei anderen unzugänglich sind. Durch die anhaltende Debatte über die Nachteile von permanenter Öffentlichkeit haben sich die Piraten in einigen wichtigen Punkten, insbesondere bei den öffentlichen Fraktionssitzungen, bereits angepasst, alles in allem aber ist die von ihnen eingeführte Transparenz immer noch erstaunlich hoch.

Die Idee, die Basis an allen politischen Entscheidungen der Partei partizipieren zu lassen, war ein faszinierendes Experiment, das aber vorerst gescheitert zu sein scheint. Die nachlassende Begeisterung der Mitglieder mit der Folge geringer Beteiligung sowie die Notwendigkeit schneller Entscheidungen, auch in Abstimmung mit anderen Fraktionen, führten zu großen Schwierigkeiten und zu Legitimationsproblemen. Gleichzeitig nahm mit der Vergrößerung des Wissensvorsprunges der Abgeordneten bei einigen die Bereitschaft ab, die für die Einbindung der Basis notwendige Arbeit zu leisten und deren Vorschläge als bindend zu akzeptieren. Die heute gelebte Praxis unterscheidet sich zwar in der Form, nicht aber in ihrer Wirkung deutlich von der anderer Fraktionen.

Das Ziel, den Fraktionsvorstand machtlos und die Fraktion damit hierarchiefrei zu belassen, ist nicht vollständig erreicht worden. Der 2013 abgewählte Vorstand versuchte, Macht zu akkumulieren, und statt formeller bildeten sich schnell informelle Hierarchien. Dennoch ist zu konstatieren, dass die Fraktion gegen die Versuche des Vorstandes, einflussreicher zu werden, bisher erfolgreich Widerstand geleistet hat. Im Vergleich zu mächtigen Vorsitzenden und klaren Hierarchien anderer Fraktionen arbeiten die Piraten immer noch weitgehend hierarchiefrei - was zwar oft schwierig, aber offensichtlich möglich ist.

Eine Fraktionsdisziplin, die die Piraten zu Beginn vehement abgelehnt haben, hat sich inzwischen etabliert. Dazu beigetragen hat weniger die Tatsache, dass die Abgeordneten gemeinsam dem Willen der Basis folgen, als vielmehr die Erkenntnis, dass bei der Vielzahl 
an komplizierten Anträgen oft die einzige Möglichkeit darin besteht, arbeitsteilig vorzugehen und seinem Fachkollegen zu folgen. Eine sachlich begründete Ablehnung der Mehrheitsmeinung der Fraktion ist in vielen Fällen rein zeitlich für den einzelnen Abgeordneten nicht zu leisten, und diejenigen, die dennoch häufiger nicht mit der Fraktion stimmen, sind schnell innerhalb der Gruppe isoliert. Auch wenn also der Fraktionsvorstand immer noch nicht vorgibt (und in Probeabstimmungen testet), wie abgestimmt werden soll, hat sich faktisch eine Art Fraktionsdisziplin herausgebildet.

Die neuen Ideen der Piratenpartei haben also in vielen Fällen die Begegnung mit der Realität nicht überstanden. Der Druck der Öffentlichkeit, aber auch der Wunsch einiger Abgeordneter, professionellere, medientauglichere Arbeit zu leisten, hat dazu ebenso beigetragen wie schlicht der arbeitsintensive Alltag im Parlament. Da diese Gründe in Zukunft nicht abnehmen werden, sind weitere Veränderungen zu erwarten. Teile der Basis und einige Abgeordnete werden das mit Schrecken, andere mit Freude sehen. Der Fokus auf die bereits erfolgten Veränderungen sollte aber nicht den Blick verstellen auf die vielen kleinen und großen Neuerungen, die die Piratenfraktion ausprobiert und beibehalten hat - in vielerlei Hinsicht ist sie tatsächlich (noch) „anders“.

\section{Innerparteiliche Willensbildung und Entscheidungsprozesse durch digitale Partizipation. Ein Praxistest des Konzepts der Liquid Democracy}

\section{Bastian Bullwinkel und Lothar Probst}

Der Hype um die Piratenpartei, der noch 2011 und 2012 die Medien, aber auch die Politikwissenschaft elektrisiert hat, ist längst vorbei. In der öffentlichen Wahrnehmung spielt die Partei trotz der Enthüllungen des Whistleblowers Edward Snowden, die eigentlich den Piraten hätten Auftrieb geben müssen, kaum noch eine Rolle. Bei den letzten Landtagswahlen und bei der Bundestagswahl 2013 landeten sie weit abgeschlagen unterhalb der Fünf-Prozent-Hürde. Trotz dieser ernüchternden Bilanz lohnt es sich, die wissenschaftliche Auseinandersetzung mit der Piratenpartei und ihrem Versprechen auf umfassende Mitgliederdemokratie fortzusetzen. Es ist schwerlich zu bestreiten, dass sie einen nachhaltigen Effekt auf die anderen Parteien und deren Formen der Mitgliederbeteiligung ausgeübt hat. Selten zuvor gab es so viele Angebote an die Mitglieder der Parteien, sich aktiv an Entscheidungsprozessen zu beteiligen. Viele Instrumente sind nicht neu und wurden auch in der Vergangenheit schon in der einen oder anderen Situation ausprobiert, aber die Beispiele sind zahlreicher geworden und zeugen von einer neuen Qualität. Sie reichen von der Urwahl der Spitzenkandidaten bei den Grünen über den Mitgliederentscheid bei der Auswahl von Programmschwerpunkten bis hin zur Mitgliederbefragung der SPD über den mit den Unionsparteien ausgehandelten Koalitionsvertrag - ein Novum in der Geschichte der Regierungsbildung, jedenfalls auf Bundesebene. ${ }^{1}$ Doch auch wenn die anderen Parteien in

1 Zu direkter Demokratie in Parteien vgl. auch den Beitrag von Rudolf Steinberg in diesem Heft der ZParl. 